HIAS-E-47

\title{
Treading a fine line: (Im)possibilities for Nash implementation with partially-honest individuals
}

Michele Lombardi

Adam Smith Business School, University of Glasgow, Glasgow, G12 8QQ, United Kingdom

Naoki Yoshihara

University of Massachusetts Amherst, Crotty Hall, 412 North Pleasant Street, Amherst, MA 01002, USA The Institute of Economic Research, Hitotsubashi University, Naka 2-1, Kuni- tachi, Tokyo 186-8603, Japan

School of Management, Kochi University of Technology, Tosayamada, Kami-city, Kochi 782-8502, Japan

July 2017

Hitotsubashi Institute for Advanced Study, Hitotsubashi University

2-1, Naka, Kunitachi, Tokyo 186-8601, Japan

tel:+81 425808604 http://hias.ad.hit-u.ac.jp/

HIAS discussion papers can be downloaded without charge from:

http://hdl.handle.net/10086/27202

https://ideas.repec.org/s/hit/hiasdp.html

All rights reserved. 


\title{
Treading a fine line: (Im)possibilities for Nash implementation with partially-honest individuals*
}

\author{
M. Lombardi ${ }^{\dagger}$ \\ University of Glasgow
}

\author{
N. Yoshihara ${ }^{\ddagger}$ \\ University of Massachusetts Amherst
}

July 5, 2017

\begin{abstract}
This paper investigates the robustness of Dutta and Sen's (2012) Theorem 1 to weaker notions of truth-telling. An individual honesty standard is modeled as a subgroup of the society, including the individual herself, for which she feels truth-telling concerns. An individual $i$ is honest when she states her true preferences as well as rankings (not necessarily complete) of outcomes that are consistent with the true preferences of individuals in her honesty standard. The paper offers a necessary condition for Nash implementation, called partial-honesty monotonicity, and shows that in an independent domain of preferences that condition is equivalent to Maskin monotonicity.
\end{abstract}

JEL classification: C72; D71; D82.

Keywords: Nash implementation; partial-honesty; non-connected honesty standards, independent domain.

\footnotetext{
* Special thanks go to Luis Corchón and Hans Peters, whose comments and suggestions have led to substantial improvements in the paper. We are also grateful to an associate editor and a referee of this journal for their helpful and thoughtful comments and suggestions. They are not responsible for any remaining deficiencies.

${ }^{\dagger}$ Corresponding author: Michele Lombardi, Adam Smith Business School, University of Glasgow, Glasgow, G12 8QQ, United Kingdom, e-mail: michele.lombardi@glasgow.ac.uk.

${ }^{\ddagger}$ Department of Economics, University of Massachusetts Amherst, Crotty Hall, 412 North Pleasant Street, Amherst, MA 01002, USA; The Institute of Economic Research, Hitotsubashi University, Naka 2-1, Kunitachi, Tokyo 186-8603, Japan; and School of Management, Kochi University of Technology, Tosayamada, Kami-city, Kochi 782-8502, Japan. E-mail: nyoshihara@econs.umass.edu
} 


\section{Introduction}

The implementation problem is the problem of designing a mechanism or game form with the property that for each profile of participants' preferences, the equilibrium outcomes of the mechanism played with those preferences coincide with the recommendations that a given social choice rule (SCR) would prescribe for that profile. If that mechanism design exercise can be accomplished, the SCR is said to be implementable. The fundamental paper on implementation in Nash equilibrium is thanks to Maskin (1999; circulated since 1977), who proves that any SCR that can be Nash implemented satisfies a remarkably strong invariance condition, now widely referred to as Maskin monotonicity. Moreover, he shows that when the mechanism designer faces at least three individuals, a SCR is Nash implementable if it is Maskin monotonic and satisfies the condition of no veto-power, subsequently, Maskin's theorem. Maskin (1999) obtains his original result by means of a mechanism that requires each individual to report, besides two auxiliary data, the whole description of the state. In a preference model, this means that each participant is asked to report preferences that members of the society have (preference profile).

Since Maskin's theorem, economists have also been interested in understanding how to circumvent the limitations imposed by Maskin monotonicity by exploring the possibilities offered by approximate (as opposed to exact) implementation (Matsushima, 1988; Abreu and Sen, 1991), as well as by implementation in refinements of Nash equilibrium (Moore and Repullo, 1988; Abreu and Sen, 1990; Palfrey and Srivastava, 1991; Jackson, 1992) and by repeated implementation (Kalai and Ledyard, 1998; Lee and Sabourian, 2011; Mezzetti and Renou, 2016). One additional way around those limitations is offered by implementation with partially-honest individuals.

A partially-honest individual is an individual who deceives the mechanism designer when the truth poses some obstacle to her material well-being. Thus, she does not deceive when the truth is equally efficacious. Simply put, a partially-honest individual follows the maxim, "Do not lie if you do not have to" to serve her material interest.

In a general environment, a seminal paper on Nash implementation problems involving partially-honest individuals is Dutta and Sen (2012), whose Theorem 1 (p. 157) shows that for implementation problems involving at least three individuals and in which there is at least one partially-honest individual, the Nash implementability is assured by no veto-power. Similar positive results are uncovered in other environments by Matsushima (2008a,b), Kartik and Tercieux (2012), Kartik et al. (2014), Lombardi and Yoshihara (2016b,c), Saporiti (2014) and Ortner (2015). Thus, there are far fewer limitations for Nash implementation when there are partially-honest individuals. ${ }^{1}$

\footnotetext{
${ }^{1}$ A pioneering work on the impact of decency constraints on Nash implementation problems is Corchón and Herrero (2004). These authors propose restrictions on sets of strategies available to agents that depend
} 
As in Maskin's (1999) original result, Dutta and Sen's (2012) Theorem 1 uses a mechanism that asks participants to report, among two auxiliary data, the whole preference profile. Moreover, according to Dutta and Sen's (2012) definition of honesty, a participant's play is honest if she plays a strategy choice which is veracious in its preference profile announcement component. In this paper, we consider weaker notions of honesty and then investigate the robustness of Dutta and Sen's (2012) Theorem 1 to these notions of truth-telling.

Under a general arbitrary definition of truth-telling, the paper shows that any SCR that can be Nash implemented with partially-honest individuals satisfies a variant of Maskin monotonicity, called partial-honesty monotonicity. First, this condition requires that a deviant is able to find a set of truthful outcomes for $\theta^{\prime}$ when $x$ is one of the outcomes selected by a given SCR at state $\theta$ and the state moves from $\theta$ to $\theta^{\prime}$. Second, it prescribes that for a deviant partially-honest individual $h$ the set of truthful strategy choices for $\theta$ differs from that for $\theta^{\prime}$ if this deviant $h$ can find a truthful outcome $z$ for $\theta^{\prime}$ that is equally good to $x$ according to her ordering $R_{h}(\theta)$, under the presumption that this $x$ is one of the outcomes selected by the given SCR at state $\theta$ but is not selected at $\theta^{\prime}$, there is a monotonic change of preferences around $x$ from $\theta$ to $\theta^{\prime}$ and, moreover, this deviant $h$ can find a truthful outcome $z^{\prime}$ for $\theta^{\prime}$ that is equally good to $x$ according to her ordering $R_{h}\left(\theta^{\prime}\right)$.

The condition is always satisfied since it always allows the case that honesty means reporting the true state of the world - as in Dutta and Sen (2012). This implies that if one would like to derive a Maskin monotonicity-type condition as a necessary condition that imposes restrictions on the class of implementable SCRs, it is bound to give a weaker meaning to the notion of honesty. Clearly, its notion needs to be compatible with the implementation environment as well as be based on testable parameters.

Since the main goal of this study is to offer notions of honesty that are weaker than that employed by Dutta and Sen (2012) and then to investigate the robustness of their result to these notions, we model individual i's honesty standard, denoted by $\mathcal{S}(i)$, as a profile of (possibly non-empty) collections of ordered pairs of outcomes, one for each member of society, over which individual $i$ feels truth-telling concerns. We write $\mathcal{S}(N) \equiv(\mathcal{S}(i))_{i \in N}$ for a typical honesty standard of society.

This notion of individual $i$ 's honesty standard is flexible enough to allow the individual $i$ 's collection for individual $j$ to be empty. Our interpretation is that in this case individual $i$ does not have any truth-telling concern about individual $j$. We also adopt the view that individual $i$ concerns herself with at least her own self; that is, her own collection of ordered pairs is not empty. Also, we require that the collection over which individual $i$ feels truthtelling concerns about herself has the property that she is able to reveal truthfully her own

on the state of the world. They refer to these strategies as decent strategies and study Nash implementation problems in decent strategies. For a particular formulation of decent strategies, they are also able to circumvent the limitations imposed by Maskin monotonicity. 
complete ranking of outcomes. This requirement turned to be an indispensable condition for honesty under which a Maskin monotonicity-type condition for Nash implementation can be derived as a necessary condition (details are in Remark 2).

Thus, an individual $i$ is honest provided that she states her true preferences as well as rankings (not necessarily complete) of outcomes that are consistent with the true preferences of individuals in her honesty standard. It is worth emphasizing that this notion of truthtelling encompasses, as a special case, that of Dutta and Sen (2012).

With these notions of honesty and honesty standards, the paper shows that any SCR that can be Nash implemented with partially-honest individuals satisfies a variant of Maskin monotonicity, called $\mathcal{S}(N)$-partial-honesty monotonicity. The idea of this axiom is quite intuitive. If $x$ is one of the outcomes selected by a given SCR at state $\theta$ but is not selected when there is a monotonic change of preferences around $x$ from $\theta$ to $\theta^{\prime}$, then the rankings of outcomes in the honesty standard of a partially-honest individual has been altered by this monotonic change. This condition implies partial-honesty monotonicity - indeed, they are equivalent under some qualifications. Furthermore, it is trivially satisfied when partiallyhonest individuals concern themselves with the announcement of the whole preference profile as in Dutta and Sen (2012). However, it can have more bite when weaker notions of honesty are considered.

Indeed, in section 5, a specific type of an individual honesty standard is considered, which is modeled as a subset of individuals involved in an implementation problem. Our interpretation is that participant $i$ concerns herself with the truth-telling of individuals in her honesty standard when she plays a strategy choice. Also, this definition endorses the view that an individual concerns herself with at least her own self. Thus, an individual $i$ is truthful provided that she states the true preferences of individuals in her honesty standard. Moreover, we consider what we call non-connected honesty standards. Simply put, individual honesty standards are connected if some participant is in the honest standard of every other participant. When that is not the case, we call them non-connected honesty standards. In other words, they are non-connected if every participant is excluded from the honesty standard of another participant.

In an independent domain of preferences, where the set of the profiles of participants' preferences takes the structure of the Cartesian product of individual preferences, we show that $\mathcal{S}(N)$-partial-honesty monotonicity is equivalent to Maskin monotonicity whenever there exists at least one partially-honest individual and individuals' honesty standards are non-connected. Thus, under those hypotheses, Maskin's theorem provides an almost complete characterization of SCRs that are Nash implementable in the society with partiallyhonest individuals.

The remainder of the paper is divided into five sections. Section 2 presents the theoret- 
ical framework and outlines the implementation model. Section 3 presents partial-honesty monotonicity, with the notions of truth-telling and of an honesty standard presented in subsection 3.1. Section 4 presents $\mathcal{S}(N)$-partial-honesty monotonicity, with the equivalence result offered in section 5 . Section 6 concludes.

\section{Preliminaries}

\subsection{Basic framework}

We consider a finite set of individuals indexed by $i \in N=\{1, \cdots, n\}$, which we will refer to as a society. The set of outcomes available to individuals is $X$. The information held by the individuals is summarized in the concept of a state. Write $\Theta$ for the domain of possible states, with $\theta$ as a typical state. In the usual fashion, individual $i$ 's preferences in state $\theta$ are given by a complete and transitive binary relation, subsequently an ordering, $R_{i}(\theta)$ over the set $X$. The corresponding strict and indifference relations are denoted by $P_{i}(\theta)$ and $I_{i}(\theta)$, respectively. The preference profile in state $\theta$ is a list of orderings for individuals in $N$ that are consistent with this state and is denoted by $R_{N}(\theta)$.

We assume that the mechanism designer does not know the true state. We assume, however, that there is complete information among the individuals in $N$ and that the mechanism designer knows the preference domain consistent with the domain $\Theta$. In this paper, sometimes we identify states with preference profiles.

The goal of the mechanism designer is to implement a social choice rule (SCR) $F$ : $\Theta \rightarrow X$ where $F(\theta)$ is non-empty for any $\theta \in \Theta$. We shall refer to $x \in F(\theta)$ as an $F$ optimal outcome at $\theta$. Given that individuals will have to be given the necessary incentives to reveal the state truthfully, the mechanism designer delegates the choice to individuals according to a mechanism $\Gamma \equiv\left(\prod_{i \in N} M_{i}, g\right)$, where $M_{i}$ is the strategy space of individual $i$ and $g: M \rightarrow X$, the outcome function, assigns to every strategy profile $m \in M \equiv \prod_{i \in N} M_{i}$ a unique outcome in $X$. We shall sometimes write $\left(m_{i}, m_{-i}\right)$ for the strategy profile $m$, where $m_{-i}=\left(m_{1}, \cdots, m_{i-1}, m_{i+1}, \cdots, m_{n}\right)$.

\subsection{Intrinsic preferences for honesty}

An individual who has an intrinsic preference for truth-telling can be thought of as an individual who is torn by a fundamental conflict between her deeply and ingrained propensity to respond to material incentives and the desire to think of herself as an honest person. In this paper, the theoretical construct of the balancing act between those contradictory desires 
is based on two ideas.

First, the pair $(\Gamma, \theta)$ acts as a "context" for individuals' conflicts. The reason for this is that an individual who has an intrinsic preference for honesty can categorize her strategy choices as truthful or untruthful relative to the state $\theta$ and the mechanism $\Gamma$ designed by the mechanism designer to govern the communication with individuals. That categorization can be captured by the following notion of truth-telling correspondence:

Definition 1 For each $\Gamma$ and each individual $i \in N$, individual $i$ 's truth-telling correspondence is a (non-empty) correspondence $T_{i}^{\Gamma}: \Theta \rightarrow M_{i}$ such that, for each $\theta \in \Theta$ and $m_{i} \in T_{i}^{\Gamma}(\theta)$, the strategy choice $m_{i}$ encodes information that is consistent with the state $\theta$. Strategy choices in $T_{i}^{\Gamma}(\theta)$ will be referred to as truthful strategy choices for $\theta$.

Second, in modeling intrinsic preferences for honesty, we endorse the notion of partiallyhonest individuals introduced by Dutta and Sen (2012). First, a partially-honest individual is an individual who responds primarily to material incentives. Second, she strictly prefers to tell the truth whenever lying has no effect on her material well-being. That behavioral choice of a partially-honest individual can be modeled by introducing an individual's ordering over the strategy space $M$ which contains the information of this individual's ordering over $X$, because that individual's preference between being truthful and being untruthful is contingent upon announcements made by other individuals as well as the outcome(s) obtained from them. By following standard conventions of orderings, write $\succcurlyeq_{i}^{\Gamma, \theta}$ for individual $i$ 's ordering over $M$ in state $\theta$ whenever she is confronted with the mechanism $\Gamma$. Formally, our notion of a partially-honest individual is as follows:

Definition 2 For each $\Gamma$, individual $i \in N$ is partially-honest if for all $\theta \in \Theta$ individual $i$ 's intrinsic preference for honesty $\succcurlyeq_{i}^{\Gamma, \theta}$ on $M$ satisfies the following properties: for all $m_{-i}$ and all $m_{i}, m_{i}^{\prime} \in M_{i}$ it holds that:

(i) If $m_{i} \in T_{i}^{\Gamma}(\theta), m_{i}^{\prime} \notin T_{i}^{\Gamma}(\theta)$ and $g(m) R_{i}(\theta) g\left(m_{i}^{\prime}, m_{-i}\right)$, then $m \succ_{i}^{\Gamma, \theta}\left(m_{i}^{\prime}, m_{-i}\right)$.

(ii) In all other cases, $m \succcurlyeq_{i}^{\Gamma, \theta}\left(m_{i}^{\prime}, m_{-i}\right)$ if and only if $g(m) R_{i}(\theta) g\left(m_{i}^{\prime}, m_{-i}\right)$.

An intrinsic preference for honesty of individual $i$ is captured by the first part of the above definition, in that, for a given mechanism $\Gamma$ and state $\theta$, individual $i$ strictly prefers the strategy profile $\left(m_{i}, m_{-i}\right)$ to $\left(m_{i}^{\prime}, m_{-i}\right)$ provided that the outcome $g\left(m_{i}, m_{-i}\right)$ is at least as good as $g\left(m_{i}^{\prime}, m_{-i}\right)$ according to her ordering $R_{i}(\theta)$ and that $m_{i}$ is truthful for $\theta$ and $m_{i}^{\prime}$ is not truthful for $\theta$.

If individual $i$ is not partially-honest, this individual cares for her material well-being associated with outcomes of the mechanism and nothing else. Then, individual $i$ 's ordering over $M$ is just the transposition into space $M$ of individual $i$ 's relative ranking of outcomes. More formally: 
Definition 3 For each $\Gamma$, individual $i \in N$ is not partially-honest if for all $\theta \in \Theta$, individual $i$ 's intrinsic preference for honesty $\succcurlyeq_{i}^{\Gamma, \theta}$ on $M$ satisfies the following property:

$$
m \succcurlyeq_{i}^{\Gamma, \theta} m^{\prime} \Longleftrightarrow g(m) R_{i}(\theta) g\left(m^{\prime}\right), \quad \text { for all } m, m^{\prime} \in M \text {. }
$$

\subsection{Implementation problems}

In formalizing the mechanism designer's problem with partially-honest individuals, we first introduce an informational assumption and discuss its implications for our analysis. It is:

Assumption 1 There exists at least one partially-honest individual in the society $N$.

Thus, in our setting, the mechanism designer only knows the set $\Theta$ as well as the fact that there is at least one partially-honest individual among the individuals, but she does not know either the true state or the identity (or identities) of the partially-honest individual(s). Indeed, the mechanism designer cannot exclude any member(s) of society from being partially-honest purely on the basis of Assumption 1. Therefore, the following considerations are in order from the viewpoint of the mechanism designer.

An environment is described by two parameters, $(\theta, H)$ : a state $\theta$ and a conceivable set of partially-honest individuals $H$. We denote by $H$ a typical conceivable set of partiallyhonest individuals in $N$, with $h$ as a typical element, and by $\mathcal{H}$ the class of conceivable sets of partially-honest individuals.

A mechanism $\Gamma$ and an environment $(\theta, H)$ induce a strategic game $\left(\Gamma, \succcurlyeq^{\Gamma, \theta, H}\right)$, where:

$$
\succcurlyeq^{\Gamma, \theta, H} \equiv\left(\succcurlyeq_{i}^{\Gamma, \theta}\right)_{i \in N}
$$

is a profile of orderings over the strategy space $M$ as formulated in Definition 2 and in Definition 3. Specifically, $\succcurlyeq_{i}^{\Gamma, \theta}$ is individual $i$ 's ordering over $M$ as formulated in Definition 2 if individual $i$ is in $H$, whereas it is the individual $i$ 's ordering over $M$ as formulated in Definition 3 if individual $i$ is not in $H$.

A (pure strategy) Nash equilibrium of the strategic game $\left(\Gamma, \succcurlyeq^{\Gamma, \theta, H}\right)$ is a strategy profile $m$ such that for all $i \in N$, it holds that

$$
m \succcurlyeq_{i}^{\Gamma, \theta}\left(m_{i}^{\prime}, m_{-i}\right), \text { for all } m_{i}^{\prime} \in M_{i} .
$$

Write $N E\left(\Gamma, \succcurlyeq^{\Gamma, \theta, H}\right)$ for the set of Nash equilibrium strategies of the strategic game $\left(\Gamma, \succcurlyeq^{\Gamma, \theta, H}\right)$ and $N A\left(\Gamma, \succcurlyeq^{\Gamma, \theta, H}\right)$ for its corresponding set of Nash equilibrium outcomes. 
The following definition is to formulate the designer's Nash implementation problem involving partially-honest individuals.

Definition 4 Let Assumption 1 hold. A mechanism $\Gamma$ partially-honestly Nash implements a SCR $F: \Theta \rightarrow X$ provided that for all $\theta \in \Theta$ there exists a truth-telling correspondence $T_{i}^{\Gamma}(\theta)$ as formulated in Definition 1 for every $i \in N$ and, moreover, it holds that

$$
F(\theta)=N A\left(\Gamma, \succcurlyeq^{\Gamma, \theta, H}\right), \quad \text { for every pair }(\theta, H) \in \Theta \times \mathcal{H} \text {. }
$$

If such a mechanism exists, $F$ is said to be partially-honestly Nash implementable.

The objective of the mechanism designer is thus to design a mechanism whose Nash equilibrium outcomes coincide with $F(\theta)$ for each state $\theta$ as well as each set $H$. Note that there is no distinction between the above formulation and the standard Nash implementation problem as long as Assumption 1 is discarded.

\section{Why is a notion of truth-telling needed?}

In this section, we present a necessary condition for partially-honest implementation, which we call partial-honesty monotonicity. This condition prescribes that for a deviant partially-honest individual $h(\in H)$ the set of truthful strategy choices for $\theta$ differs from that for $\theta^{\prime}$ if this individual $h$ can find a truthful outcome $z$ for the state $\theta^{\prime}$ that is equally good to $x$ according to her ordering $R_{h}(\theta)$ and provided that $x$ is $F$-optimal at $\theta$ but not $F$-optimal at $\theta^{\prime}$, that there is a monotonic change of preferences around $x$ from $\theta$ to $\theta^{\prime}$ and that this deviant $h$ can find a truthful outcome $z^{\prime}$ for $\theta^{\prime}$ that is equally good to $x$ according to her ordering $R_{h}\left(\theta^{\prime}\right)$. Let us formalize the condition as follows. Given a state $\theta$, an individual $i$, and an outcome $x \in X$, the weak lower contour set of $R_{i}(\theta)$ at $x$ is $L_{i}(\theta, x) \equiv\left\{x^{\prime} \in X \mid x R_{i}(\theta) x^{\prime}\right\}$; and the indifferent contour set of $R_{i}(\theta)$ at $x$ is $I_{i}(\theta, x) \equiv\left\{x^{\prime} \in X \mid x I_{i}(\theta) x^{\prime}\right\}$. Therefore:

Definition 5 A SCR $F: \Theta \rightarrow X$ is partial-honesty monotonic provided that for each individual $i \in N$, there exist a (non-empty) set $A_{i}$ and a (non-empty) correspondence $T_{i}$ : $\Theta \rightarrow A_{i}$ such that for all $H \in \mathcal{H}$ and all $\theta, \theta^{\prime} \in \Theta$, if $x \in F(\theta) \backslash F\left(\theta^{\prime}\right)$ and $L_{i}(\theta, x) \subseteq L_{i}\left(\theta^{\prime}, x\right)$ for each $i \in N$, then for at least one $h \in H$ there exists a (non-empty) set $S_{h}\left(\theta^{\prime} ; x, \theta\right) \subseteq$ $L_{h}(\theta, x)$ such that $S_{h}\left(\theta^{\prime} ; x, \theta\right) \cap I_{h}\left(\theta^{\prime}, x\right) \neq \varnothing$ holds, and:

$$
S_{h}\left(\theta^{\prime} ; x, \theta\right) \cap I_{h}(\theta, x) \neq \varnothing \Longrightarrow T_{h}(\theta) \neq T_{h}\left(\theta^{\prime}\right) .
$$

Let us give an intuitive explanation of the set $S_{i}\left(\theta^{\prime} ; x, \theta\right)$. Suppose that $F$ is partiallyhonestly Nash implementable. Suppose that $x=g(m)$ is $F$-optimal at $\theta$, that is, $x \in F(\theta)$. 
Whilst the set $g\left(M_{i}, m_{-i}\right)$ represents the set of outcomes that individual $i$ can generate by varying her own strategy, keeping the other individuals' equilibrium strategy choices fixed at $m_{-i}$, the set $S_{i}\left(\theta^{\prime} ; x, \theta\right)=g\left(T_{i}^{\Gamma}\left(\theta^{\prime}\right), m_{-i}\right)$ represents the set of outcomes that this individual can attain by playing truthful strategy choices for $\theta^{\prime}$ when the state moves from $\theta$ to $\theta^{\prime}$, keeping the other individuals' equilibrium strategy choices fixed at $m_{-i}$. Given this idea of the set of $S_{i}\left(\theta^{\prime} ; x, \theta\right)$, we refer to elements of $S_{i}\left(\theta^{\prime} ; x, \theta\right)$ as truthful outcomes for individual $i$ at the state $\theta^{\prime}$ when the state moves from $\theta$ to $\theta^{\prime}$ and $x$ is an $F$-optimal outcome at $\theta$.

Our first main result is that only partial-honesty monotonic SCRs are partially-honestly Nash implementable.

Theorem 1 Let Assumption 1 be given. A SCR $F: \Theta \rightarrow X$ is partial-honesty monotonic if it is partially-honestly Nash implementable.

Proof. Let Assumption 1 be given. Suppose that $\Gamma \equiv(M, g)$ partially-honestly Nash implements the SCR $F: \Theta \rightarrow X$. Thus, for all $\bar{\theta} \in \Theta$ there exists a (non-empty) truthtelling correspondence $T_{i}^{\Gamma}(\bar{\theta})$ as formulated in Definition 1 for every $i \in N$ and, moreover, it holds that $F(\bar{\theta})=N A\left(\Gamma, \succcurlyeq^{\Gamma, \bar{\theta}, H}\right)$ for every pair $(\bar{\theta}, H) \in \Theta \times \mathcal{H}$. Thus, for each individual $i$, let us define the set $A_{i}$ by $A_{i}=M_{i}$ and the correspondence $T_{i}$ by $T_{i}=T_{i}^{\Gamma}$.

Fix any $(\theta, H) \in \Theta \times \mathcal{H}$ such that $x \in F(\theta)$. Thus, there is $m \in N E\left(\Gamma, \succcurlyeq^{\Gamma, \theta, H}\right)$ such that $g(m)=x$.

Consider any state $\theta^{\prime} \in \Theta$ such that

$$
\text { for all } i \in N \text { and all } x^{\prime} \in X: x R_{i}(\theta) x^{\prime} \Longrightarrow x R_{i}\left(\theta^{\prime}\right) x^{\prime} \text {. }
$$

If there exists an individual $i \in N$ such that $g\left(m_{i}^{\prime}, m_{-i}\right) P_{i}\left(\theta^{\prime}\right) g(m)$, then, from (1),

$$
g\left(m_{i}^{\prime}, m_{-i}\right) P_{i}(\theta) g(m)
$$

a contradiction of the fact that $m \in N E\left(\Gamma, \succcurlyeq^{\Gamma, \theta, H}\right)$. Therefore, we conclude that

$$
\text { for all } i \in N \text { and all } m_{i}^{\prime} \in M_{i}: g(m) R_{i}\left(\theta^{\prime}\right) g\left(m_{i}^{\prime}, m_{-i}\right) \text {. }
$$

Suppose that $x \notin F\left(\theta^{\prime}\right)$. Then, the strategy profile $m$ is not a Nash equilibrium for $\left(\Gamma, \succcurlyeq^{\Gamma, \theta^{\prime}, H}\right)$; that is, there exists an individual $i \in N$ who can find a strategy choice $m_{i}^{\prime} \in M_{i}$ such that $\left(m_{i}^{\prime}, m_{-i}\right) \succ_{i}^{\Gamma, \theta^{\prime}} m$. Given that (2) holds, it must be the case that $i \in H$. From part (i) of Definition 2 we conclude, therefore, that

$$
m_{i} \notin T_{i}^{\Gamma}\left(\theta^{\prime}\right) \text { and } m_{i}^{\prime} \in T_{i}^{\Gamma}\left(\theta^{\prime}\right)
$$


and that

$$
g\left(m_{i}^{\prime}, m_{-i}\right) R_{i}\left(\theta^{\prime}\right) g(m) .
$$

For this $i \in H$, let us define the set $S_{i}\left(\theta^{\prime} ; x, \theta\right)$ by

$$
S_{i}\left(\theta^{\prime} ; x, \theta\right)=g\left(T_{i}^{\Gamma}\left(\theta^{\prime}\right), m_{-i}\right) .
$$

It is plain that this set is not empty - since $T_{i}^{\Gamma}\left(\theta^{\prime}\right)$ is not empty - and that $S_{i}\left(\theta^{\prime} ; x, \theta\right) \subseteq$ $L_{i}(\theta, x)$. Moreover, by (2) and (4), individual $i$ is indifferent between $g(m)$ and $g\left(m_{i}^{\prime}, m_{-i}\right)$, and by (3) and (5), $g\left(m_{i}^{\prime}, m_{-i}\right)$ is an element of $S_{i}\left(\theta^{\prime} ; x, \theta\right)$. Thus, $g\left(m_{i}^{\prime}, m_{-i}\right) \in S_{i}\left(\theta^{\prime} ; x, \theta\right) \cap$ $I_{i}\left(\theta^{\prime}, x\right)$, which implies $S_{i}\left(\theta^{\prime} ; x, \theta\right) \cap I_{i}\left(\theta^{\prime}, x\right) \neq \varnothing$.

To show the remaining property, we use a proof by contrapositive here. Assume that $T_{i}^{\Gamma}\left(\theta^{\prime}\right)=T_{i}^{\Gamma}(\theta)$. Suppose that there is a $w \in X$ such that $w \in S_{i}\left(\theta^{\prime} ; x, \theta\right) \cap I_{i}(\theta, x)$. Thus, by definition of $S_{i}\left(\theta^{\prime} ; x, \theta\right)$ in (5), it follows that there exists $m_{i}^{\prime \prime} \in T_{i}^{\Gamma}\left(\theta^{\prime}\right)=T_{i}^{\Gamma}(\theta)$ such that $g\left(m_{i}^{\prime \prime}, m_{-i}\right)=w$ and that

$$
g\left(m_{i}^{\prime \prime}, m_{-i}\right) I_{i}(\theta) g(m) .
$$

Furthermore, since $T_{i}^{\Gamma}\left(\theta^{\prime}\right)=T_{i}^{\Gamma}(\theta)$, it follows from (3) that $m_{i} \notin T_{i}^{\Gamma}(\theta)$. Given that (6) holds, from part (i) of Definition 2 we conclude, therefore, that $m$ is not a Nash equilibrium for $\left(\Gamma, \succcurlyeq^{\Gamma, \theta, H}\right)$, which is a contradiction. Then, the intersection $S_{i}\left(\theta^{\prime} ; x, \theta\right) \cap I_{i}(\theta, x)$ needs to be empty, and so $F$ is partial-honesty monotonic.

It is worth emphasizing that the above condition does not impose any restriction on the class of SCRs that are partially-honestly Nash implementable. This is so because for any $\theta, \theta^{\prime} \in \Theta$ with $x \in F(\theta) \backslash F\left(\theta^{\prime}\right)$, the set $S_{i}\left(\theta^{\prime} ; x, \theta\right)$ can be defined as $S_{i}\left(\theta^{\prime} ; x, \theta\right)=L_{i}(\theta, x)$ for each $i \in N$, and there always exists an individual $i$ 's correspondence $T_{i}$ defined by $T_{i}(\theta)=\{\theta\}$ for each $\theta \in \Theta$, for each $i \in N$, under which $T_{i}(\theta) \neq T_{i}\left(\theta^{\prime}\right)$ always holds for any $\theta, \theta^{\prime} \in \Theta$ with $\theta \neq \theta^{\prime}$. This is consistent with Theorem 1 of Dutta and Sen (2012), according to which the partially-honest Nash implementability is assured by no veto-power when to be honest means to report the true state of the world.

Corollary 1 Every SCR $F: \Theta \rightarrow X$ is partial-honesty monotonic.

However, the condition may be stringent when for each individual $i$, the value of the correspondence $T_{i}$ is constrained by a weaker notion of honesty: for any admissible corrrespondence profile $\left(T_{i}\right)_{i \in N}$, there exist $\theta \in \Theta$ and $j \in N$ such that $T_{j}(\theta) \neq\{\theta\}$. This will be the subject of what follows below. 


\subsection{Truth-telling and honesty standards}

The main practical aim of adopting an axiomatic approach to Nash implementation theory is to distinguish between implementable and non-implementable SCRs. Thus, the importance and usefulness of a necessary condition for implementation relies on its testability. This means that it has to involve only observable parameters such as preferences and outcomes.

Furthermore, the seminal result of Dutta and Sen (2012) requires that an individual's report is truthful when it conveys the true preferences of individuals. However, since the goal of this paper is to check the robustness of this seminal result to weaker notions of truthtelling, we adopt the view that an individual $i$ is truthful when she states her true preference as well as rankings (not necessarily complete) of outcomes that are consistent with the true preferences of individuals for whom this individual $i$ feels truth-telling concerns.

Let us formalize our notions of truth-telling as well as individuals' honesty standards. Let the family $\mathcal{X}$ have as elements all non-empty subsets of the space $X \times X$ as well as the set whose element is the empty set. As usual, let us denote by $\mathcal{X}^{n}$ the $n$-fold Cartesian product of the family $\mathcal{X}$.

An honesty standard of individual $i$, denoted by $\mathcal{S}(i) \equiv\left(\mathcal{S}_{j}(i)\right)_{j \in N}$, is an element of $\mathcal{X}^{n}$ (that is, $\mathcal{S}(i) \in \mathcal{X}^{n}$ ). Whilst our interpretation of the set $\mathcal{S}_{j}(i)=\left\{\varnothing^{j}\right\}$ is that individual $i$ does not have any truth-telling concern about individual $j$, our interpretation of the set $\mathcal{S}_{j}(i) \neq\left\{\varnothing^{j}\right\}$ is that individual $i$ concerns herself about individual $j$ and the set $\mathcal{S}_{j}(i)$ represents the collection of ordered pairs over which this $i$ feels truth-telling concerns - when she plays a strategy choice. An honesty standard of society is a list of honesty standards for all members of society. Write $\mathcal{S}(N) \equiv(\mathcal{S}(i))_{i \in N}$ for a typical honesty standard of society.

We adopt the view that individual $i$ concerns herself with at least her own self; that is, $\mathcal{S}_{i}(i) \neq\left\{\varnothing^{i}\right\}$. Moreover, the collection $\mathcal{S}_{i}(i)$ of ordered pairs over which she feels truthtelling concerns has the property that this $i$ is able to reveal truthfully her complete ranking of outcomes; formally, we adopt the view that $\mathcal{S}(i)$ is an honesty standard of individual $i$ provided that

$$
\mathcal{S}_{i}(i) \cap R_{i}(\theta)=R_{i}(\theta) \text { for all } \theta \in \Theta .
$$

The indispensability of these requirements for the honesty standard of individual $i$ will be discussed in Remark 2. Our interpretation of these requirements is that individual $i$, to view herself as an honest person, has at least to concern herself with the truth-telling of her own preference ordering. This also means that individual $i$ may display an honesty standard which allows her to hide partially or totally other individuals' rankings over outcomes without that being harmful to her self view as an honest person.

Let us observe that this formulation of an honesty standard satisfies important proper- 
ties. First, it does not depend on the current state of the world. Second, it is also independent of the social objectives that society or its representatives want to achieve. Last but not least, our formulation of honesty standards do not hinge on the existence of any mechanism. All in all, it has the property to be formulated only on observable parameters.

We are now in a position to state our notion of truth-telling. Formally, for a given state $\theta$ and individual $i$ 's honesty standard $\mathcal{S}(i)$, to save notation we write $R_{N}(\theta) \cap \mathcal{S}(i)$ for $R_{j}(\theta) \cap \mathcal{S}_{j}(i)$ for each individual $j$. Thus:

Definition 6 For each $\Gamma$ and each individual $i \in N$ with an honesty standard $\mathcal{S}(i)$ satisfying the requirement in (7), individual $i$ 's truth-telling correspondence is a (non-empty) correspondence $T_{i}^{\Gamma}(\cdot ; \mathcal{S}(i)): \Theta \rightarrow M_{i}$ with the property that for any two states $\theta$ and $\theta^{\prime}$, it holds that

$$
T_{i}^{\Gamma}(\theta ; \mathcal{S}(i))=T_{i}^{\Gamma}\left(\theta^{\prime} ; \mathcal{S}(i)\right) \Longleftrightarrow R_{N}(\theta) \cap \mathcal{S}(i)=R_{N}\left(\theta^{\prime}\right) \cap \mathcal{S}(i)
$$

Strategy choices in $T_{i}^{\Gamma}(\theta ; \mathcal{S}(i))$ will be referred to as truthful strategy choices for $\theta$ according to $\mathcal{S}(i)$.

According to the above definition, in a state $\theta$, every truthful strategy choice of individual $i$ is to encode information of individuals' rankings of outcomes that are consistent with the profile of individuals' orderings at the state $\theta$. Moreover, if in two different states, say $\theta$ and $\theta^{\prime}$, it holds that for each individual $j$, the set of ordered pairs in $\mathcal{S}_{j}(i)$ that are consistent with individual $j$ 's ordering at $\theta$ is identical to the set of ordered pairs that are consistent with individual $j$ 's ordering at $\theta^{\prime}$ (that is, $R_{j}(\theta) \cap \mathcal{S}_{j}(i)=R_{j}\left(\theta^{\prime}\right) \cap \mathcal{S}_{j}(i)$ ), then the sets of individual $i$ 's truthful strategy choices for those two states need to be identical according to her honesty standard $\mathcal{S}(i)$.

The above definition of truth-telling imposes a mild restriction on the class of truthtelling correspondences and, perhaps more interestingly, it represents a minimal notion of honesty that one can formulate in our general environment. It is vital to emphasize here that our notion of veracity encompasses, as a special case, that of Dutta and Sen (2012) when each individual $i$ 's honesty standard $\mathcal{S}(i)$ is such that $\mathcal{S}_{j}(i) \cap R_{j}(\theta)=R_{j}(\theta)$ for every individual $j$ and every state $\theta$.

\section{4. $\mathcal{S}(N)$-partial-honesty monotonicity}

In this section, we discuss a condition, called $\mathcal{S}(N)$-partial-honesty monotonicity, that is necessary for the partially-honest Nash implementation when the honesty standard of society is prescribed by $\mathcal{S}(N)$. We also show that this condition implies partial-honesty 
monotonicity. Moreover, for any given honesty standard of society summarized in $\mathcal{S}(N)$, the two conditions are equivalent when each individual $i$ 's correspondence $T_{i}$ presented in Definition 5 is required to be consistent with $\mathcal{S}(N)$ (see below).

A condition that is central to the implementation of SCRs in Nash equilibrium is Maskin monotonicity. This condition says that if an outcome $x$ is $F$-optimal at the state $\theta$, and this $x$ does not strictly fall in preference for anyone when the state is changed to $\theta^{\prime}$, then $x$ must remain an $F$-optimal outcome at $\theta^{\prime}$. Let us formalize that condition as follows:

Definition 7 A SCR $F: \Theta \rightarrow X$ is Maskin monotonic provided that for all $x \in X$ and all $\theta, \theta^{\prime} \in \Theta$, if $x \in F(\theta)$ and $L_{i}(\theta, x) \subseteq L_{i}\left(\theta^{\prime}, x\right)$ for all $i \in N$, then $x \in F\left(\theta^{\prime}\right)$.

An equivalent statement of Maskin monotonicity stated above follows the reasoning that if $x$ is $F$-optimal at $\theta$ but not $F$-optimal at $\theta^{\prime}$, then the outcome $x$ must have fallen strictly in someone's ordering at the state $\theta^{\prime}$ in order to break the Nash equilibrium via some deviation. Therefore, there must exist some (outcome-)preference reversal if an equilibrium strategy profile at $\theta$ is to be broken at $\theta^{\prime}$.

Our variant of Maskin monotonicity for Nash implementation problems involving partiallyhonest individuals when the standard of honesty in a society is represented by $\mathcal{S}(N)$ can be formulated as follows:

Definition 8 A SCR $F: \Theta \rightarrow X$ is $\mathcal{S}(N)$-partial-honesty monotonic given the standard $\mathcal{S}(N)$ (satisfying the requirement in (7) for each individual $i$ ) provided that for all $x \in X$, all $H \in \mathcal{H}$ and all $\theta, \theta^{\prime} \in \Theta$, if $x \in F(\theta) \backslash F\left(\theta^{\prime}\right)$ and $L_{i}(\theta, x) \subseteq L_{i}\left(\theta^{\prime}, x\right)$ for all $i \in N$, then there exists at least one $h \in H$ such that $R_{N}(\theta) \cap \mathcal{S}(h) \neq R_{N}\left(\theta^{\prime}\right) \cap \mathcal{S}(h)$.

This says that if $x$ is $F$-optimal at $\theta$ but not $F$-optimal at $\theta^{\prime}$ and, moreover, there is a monotonic change of preferences around $x$ from $\theta$ to $\theta^{\prime}$ (that is, whenever $x R_{i}(\theta) x^{\prime}$, one has that $\left.x R_{i}\left(\theta^{\prime}\right) x^{\prime}\right)$, then the rankings of outcomes in the honesty standard of a partiallyhonest individual $h$ has been altered by this monotonic change (that is, $R_{N}(\theta) \cap \mathcal{S}(h) \neq$ $\left.R_{N}\left(\theta^{\prime}\right) \cap \mathcal{S}(h)\right)$. Stated in the contrapositive, this says that if $x$ is $F$-optimal at $\theta$ and there is a monotonic change of preferences around $x$ from $\theta$ to $\theta^{\prime}$ and, moreover, the rankings of outcomes in the honesty standard of every partially-honest individual $h$ in $H$ has not been altered by this monotonic change, then $x$ must continue to be one of the outcomes selected by $F$ at the state $\theta^{\prime}$.

Remark 1 Note that if each individual $i$ 's honesty standard $\mathcal{S}(i)$ is such that $\mathcal{S}_{j}(i) \cap$ $R_{j}(\theta)=R_{j}(\theta)$, for every individual $j$ and every state $\theta$, and if $x$ is $F$-optimal at $\theta$ but not $F$-optimal at $\theta^{\prime}$ and it happens that the lower contour sets of preferences at $x$ are nested for every agent across the two environments, then one has that $R_{N}(\theta) \neq R_{N}\left(\theta^{\prime}\right)$. Thus, any 
SCR is $\mathcal{S}(N)$-partial-honesty monotonic whenever the honesty standard of society is that studied by Dutta and Sen (2012).

The above condition is necessary for partially-honest Nash implementation. This is because if $x$ is $F$-optimal at $\theta$ but not $F$-optimal at $\theta^{\prime}$ and, moreover, the outcome $x$ has not fallen strictly in any individual's ordering at the state $\theta^{\prime}$, then only a partially-honest individual in the given conceivable set $H$ can break the Nash equilibrium via a unilateral deviation. Therefore, there must exist a partially-honest individual $h \in H$ whose equilibrium strategy to attain $x$ at $(\theta, \mathcal{S}(N), H)$ is not a truthful strategy choice at $\left(\theta^{\prime}, \mathcal{S}(N), H\right)$. This means that $R_{N}(\theta) \cap \mathcal{S}(h) \neq R_{N}\left(\theta^{\prime}\right) \cap \mathcal{S}(h)$, according to Definition 6 .

Note that the above definitions of partially-honest individuals (that is, Definition 2) as well as of partially-honest Nash implementation can be easily adapted to the environments with honesty standards. Now, a mechanism $\Gamma$ and an environment with honesty standards $(\theta, \mathcal{S}(N), H)$ induces a strategic game $\left(\Gamma, \succcurlyeq^{\Gamma, \theta, \mathcal{S}(N), H}\right)$, where:

$$
\succcurlyeq^{\Gamma, \theta, \mathcal{S}(N), H} \equiv\left(\succcurlyeq_{i}^{\Gamma, \theta, \mathcal{S}(i)}\right)_{i \in N}
$$

is a profile of orderings over the strategy space $M$ as formulated in Definition 2 and in Definition 3 where the truth-telling correspondence of individual $i$ is that provided in Definition 6. Moreover, our notion of implementation in an environment with honesty standards can be stated as follows:

Definition 9 Let Assumption 1 be given. Let the honesty standard of society be summarized in $\mathcal{S}(N)$. A mechanism $\Gamma$ partially-honestly Nash implements a SCR $F: \Theta \rightarrow X$ provided that for all $\theta \in \Theta$ and $H \in \mathcal{H}$ there exists for any $h \in H$ a truth-telling correspondence $T_{h}^{\Gamma}(\theta ; \mathcal{S}(h))$ as formulated in Definition 6 and, moreover, it holds that $F(\theta)=N A\left(\Gamma, \succcurlyeq^{\Gamma, \theta, \mathcal{S}(N), H}\right)$. If such a mechanism exists, $F$ is said to be partially-honestly Nash implementable.

Therefore, our second main result can be stated as follows:

Theorem 2 Let Assumption 1 be given. Let the honesty standard of society be summarized in $\mathcal{S}(N)$. A SCR $F: \Theta \rightarrow X$ is $\mathcal{S}(N)$-partial-honesty monotonic given the standard $\mathcal{S}(N)$ if it is partially-honestly Nash implementable.

Proof. Let Assumption 1 be given. Let the honesty standard of society be summarized in $\mathcal{S}(N)$. Suppose that $\Gamma \equiv(M, g)$ partially-honestly Nash implements the SCR $F: \Theta \rightarrow X$. This proof is almost identical to the proof of Theorem 1 up to equation (4). We thus urge the reader to consult that part of the proof before reading what follows. 
Therefore, the premises of the condition are met. Moreover, note that (2) and (4) jointly imply that

$$
g\left(m_{i}^{\prime}, m_{-i}\right) I_{i}\left(\theta^{\prime}\right) g(m) .
$$

We show that $R_{N}(\theta) \cap \mathcal{S}(i) \neq R_{N}\left(\theta^{\prime}\right) \cap \mathcal{S}(i)$. Assume, to the contrary, that

$$
\text { for all } h \in H: R_{N}(\theta) \cap \mathcal{S}(h)=R_{N}\left(\theta^{\prime}\right) \cap \mathcal{S}(h) \text {. }
$$

Definition 6 implies that

$$
\text { for all } h \in H: T_{h}^{\Gamma}(\theta ; \mathcal{S}(h))=T_{h}^{\Gamma}\left(\theta^{\prime} ; \mathcal{S}(h)\right) \text {. }
$$

Given that (3) is equivalent to

$$
m_{i} \notin T_{i}^{\Gamma}\left(\theta^{\prime} ; \mathcal{S}(i)\right) \text { and } m_{i}^{\prime} \in T_{i}^{\Gamma}\left(\theta^{\prime} ; \mathcal{S}(i)\right)
$$

(11) implies that

$$
m_{i} \notin T_{i}^{\Gamma}(\theta ; \mathcal{S}(i)) \text { and } m_{i}^{\prime} \in T_{i}^{\Gamma}(\theta ; \mathcal{S}(i)) .
$$

Furthermore, given that requirement (7) holds, (9) and (10) jointly imply that $R_{i}(\theta)=R_{i}\left(\theta^{\prime}\right)$ and that

$$
g\left(m_{i}^{\prime}, m_{-i}\right) I_{i}(\theta) g(m) .
$$

Given (12) and (13) and the fact that $i \in H$, Definition 2 implies that $\left(m_{i}^{\prime}, m_{-i}\right) \succ_{i}^{\Gamma, \theta, \mathcal{S}(i)} m$, which is a contradiction of the fact that $m \in N E\left(\Gamma, \succcurlyeq^{\Gamma, \theta, \mathcal{S}(N), H}\right)$. Thus, $F$ is $\mathcal{S}(N)$-partialhonesty monotonic given the honesty standard $\mathcal{S}(N)$.

Remark 2 The necessity of the $\mathcal{S}(N)$-partial-honesty monotonicity for partially-honest Nash implementation relies on the requirement that every individual to view herself as an honest person has at least to concern herself with the truth-telling of her own preference ordering (that is, on the requirement in (7)). Indeed, if this requirement fails to hold, then it would not be possible to conclude in (13) that individual $i$ judges the outcomes $g\left(m_{i}^{\prime}, m_{-i}\right)$ and $g(m)$ as equally good according to her preference ordering at the state $\theta$, and so it would not be possible to conclude that this $i$ can break the Nash equilibrium strategy profile $m$ at the state $\theta$ via a unilateral deviation. From this perspective, we view this requirement as a sufficient condition for honesty under which a Maskin monotonicity-type condition can be derived as a necessary condition for partially-honest Nash implementation.

Furthermore, $\mathcal{S}(N)$-partial-honesty monotonicity implies partial-honesty monotonicity, as we show next. 
Theorem 3 If a SCR $F: \Theta \rightarrow X$ is $\mathcal{S}(N)$-partial-honesty monotonic, then it is partialhonesty monotonic.

Proof. Assume that the SCR $F: \Theta \rightarrow X$ is $\mathcal{S}(N)$-partial-honesty monotonic. We show that it is partial-honesty monotonic too. For each individual $i$, let us define the set $A_{i}$ by $A_{i}=\mathcal{X}^{n}$ and the correspondence $T_{i}: \Theta \rightarrow A_{i}$ by $T_{i}(\theta)=R_{N}(\theta) \cap \mathcal{S}(i)$, for all $\theta \in \Theta$. Furthermore, for each individual $i$, for each $\theta$ such that $x \in F(\theta)$ and each $\theta^{\prime}$, let us define the set $S_{i}\left(\theta^{\prime} ; x, \theta\right)$ by $S_{i}\left(\theta^{\prime} ; x, \theta\right)=L_{i}(\theta, x)$. Thus, for each individual $i, S_{i}\left(\theta^{\prime} ; x, \theta\right) \cap I_{i}\left(\theta^{\prime}, x\right) \neq \varnothing$, and $x \in S_{i}\left(\theta^{\prime} ; x, \theta\right) \cap I_{i}(\theta, x)$ holds for all $\theta$ and $\theta^{\prime}$ with $x \in F(\theta)$.

Fix any $H \in \mathcal{H}$. Take any $\theta$ and $\theta^{\prime}$ such that $x \in F(\theta) \backslash F\left(\theta^{\prime}\right)$. Furthermore, let us suppose that $L_{i}(\theta, x) \subseteq L_{i}\left(\theta^{\prime}, x\right)$ for each individual $i$. Since $F$ is $\mathcal{S}(N)$-partial-honesty monotonic, it implies that $R_{N}(\theta) \cap \mathcal{S}(h) \neq R_{N}\left(\theta^{\prime}\right) \cap \mathcal{S}(h)$ for at least one $h \in H$. This means that $T_{h}(\theta) \neq T_{h}\left(\theta^{\prime}\right)$ for at least one $h \in H$, as we sought. Thus, $F$ is partial-honesty monotonic.

Indeed, for any given honesty standard of society summarized in $\mathcal{S}(N)$, the two conditions are equivalent when every individual $i$ 's correspondence $T_{i}$ is required to be consistent with $\mathcal{S}(N)$ in the following sense: The profile of correspondences $\left(T_{i}\right)_{i \in N}$, specified by partially-honest monotonicity, is consistent with $\mathcal{S}(N)$ if and only if for any $\theta$ and $\theta^{\prime}$ in $\Theta$, it holds that

$$
T_{i}(\theta)=T_{i}\left(\theta^{\prime}\right) \Longleftrightarrow R_{N}(\theta) \cap \mathcal{S}(i)=R_{N}\left(\theta^{\prime}\right) \cap \mathcal{S}(i) \text {, for each } i \in N \text {. }
$$

Formally:

Corollary 2 Let the honesty standard of society be summarized in $\mathcal{S}(N)$. Let the profile of correspondences $\left(T_{i}\right)_{i \in N}$, specified by partially-honest monotonicity, be consistent with $\mathcal{S}(N)$. Then, partial-honesty monotonicity is equivalent to $\mathcal{S}(N)$-partial-honesty monotonicity.

Proof. By Theorem 3, $\mathcal{S}(N)$-partial-honesty monotonicity implies partial-honesty monotonicity. Therefore, it is sufficient to show the converse relation. To this end, take any $\theta$ and $\theta^{\prime}$ such that $x \in F(\theta) \backslash F\left(\theta^{\prime}\right)$ and suppose that $L_{i}(\theta, x) \subseteq L_{i}\left(\theta^{\prime}, x\right)$ for each individual $i$. Let this $F$ satisfy partial-honesty monotoniciy with the requirement that every individual $i$ 's correspondence $T_{i}$ is consistent with $\mathcal{S}(N)$. Suppose that $F$ does not satisfy $\mathcal{S}(N)$-partialhonest monotonicity. That is, suppose that, for any $i \in N, R_{N}(\theta) \cap \mathcal{S}(i)=R_{N}\left(\theta^{\prime}\right) \cap \mathcal{S}(i)$. By (14), it follows that $T_{i}(\theta)=T_{i}\left(\theta^{\prime}\right)$ for each $i \in N$. Partial-honesty monotonicity implies that for at least one $h \in H$, it holds that $S_{h}\left(\theta^{\prime} ; x, \theta\right) \cap I_{h}\left(\theta^{\prime}, x\right)$ is not empty. Since $T_{h}(\theta)=T_{h}\left(\theta^{\prime}\right)$, partial-honesty monotonicity also implies that $S_{h}\left(\theta^{\prime} ; x, \theta\right) \cap I_{h}(\theta, x)$ is empty. However, since $R_{N}(\theta) \cap \mathcal{S}(h)=R_{N}\left(\theta^{\prime}\right) \cap \mathcal{S}(h)$, requirement (7) implies that 
$R_{h}(\theta)=R_{h}\left(\theta^{\prime}\right)$, and so $I_{h}\left(\theta^{\prime}, x\right)=I_{h}(\theta, x)$. Since $S_{h}\left(\theta^{\prime} ; x, \theta\right) \cap I_{h}\left(\theta^{\prime}, x\right)$ is not empty, it follows that $S_{h}\left(\theta^{\prime} ; x, \theta\right) \cap I_{h}(\theta, x)$ is not empty, which is a contradiction. Thus, $F$ satisfies $\mathcal{S}(N)$-partial-honest monotonicity.

\section{Equivalence result}

The classic paper on Nash implementation theory is Maskin (1999), which shows that where the mechanism designer faces a society involving at least three individuals, a SCR is Nash implementable if it is Maskin monotonic and satisfies the auxiliary condition of no veto-power. $^{2}$

The condition of no veto-power says that if an outcome is at the top of the preferences of all individuals but possibly one, then it should be chosen irrespective of the preferences of the remaining individual; that individual cannot veto it. Formally: ${ }^{3}$

Definition 10 A SCR $F: \Theta \rightarrow X$ satisfies no veto-power provided that for all $\theta \in \Theta$ and all $x \in X$, if

$$
\left|\left\{i \in N \mid X \subseteq L_{i}(\theta, x)\right\}\right| \geq n-1
$$

then $x \in F(\theta)$.

Proposition 1 (Maskin's Theorem, 1999) If $n \geq 3$ and $F: \Theta \rightarrow X$ is a SCR satisfying Maskin monotonicity and no veto-power, then it is Nash implementable.

In a general environment such as that considered here, a seminal paper on Nash implementation problems involving partially-honest individuals is Dutta and Sen (2012). It shows that for Nash implementation problems involving at least three individuals and in which there is at least one partially-honest individual, the Nash implementability is assured by no veto-power (Dutta and Sen, 2012; p. 157). From the perspective of this paper, Dutta-Sen's notion of truth-telling and their Theorem 1 can be formally restated as follows.

We have already mentioned that our notion of truth-telling encompasses, as a special case, that of Dutta and Sen (2012) provided that each individual $i$ 's honesty standard $\mathcal{S}(i)$ is such that $\mathcal{S}_{j}(i) \cap R_{j}(\theta)=R_{j}(\theta)$ for every individual $j$ and every state $\theta$. As a generalization of Dutta and Sen's (2012) honesty standard, let us consider a specific type of an honesty

\footnotetext{
${ }^{2}$ Moore and Repullo (1990), Dutta and Sen (1991), Sjöström (1991) and Lombardi and Yoshihara (2013) refined Maskin's theorem by providing necessary and sufficient conditions for an SCR to be implementable in (pure strategies) Nash equilibrium. For an introduction to the theory of implementation see Jackson (2001), Maskin and Sjöström (2002) and Serrano (2004).

${ }^{3}$ For any finite set $S,|S|$ denotes the cardinality of $S$.
} 
standard $\mathcal{S}(i)$ of each individual $i$ such that:

for any $j \in N, \mathcal{S}_{j}(i) \neq\left\{\varnothing^{j}\right\}$ implies $\mathcal{S}_{j}(i) \cap R_{j}(\theta)=R_{j}(\theta)$ for every state $\theta$.

Such a type of honesty standard ensures the existence of a subgroup of society, denoted by $S(i)$, such that $j \in S(i)$ if and only if $\mathcal{S}_{j}(i) \cap R_{j}(\theta)=R_{j}(\theta)$ for every state $\theta$. In this section, we focus our attention to this type of honesty standards for all individuals. To ease notation, in what follows we can denote an honesty standard of individual $i$ by $S(i)$. Thus, given a state $\theta, R_{S(i)}(\theta)$ is a list of orderings consistent with $\theta$ for individuals in the honesty standard $S(i)$ of individual $i$. Our interpretation is that participant $i$ concerns herself with the truth-telling of preferences of individuals in her honesty standard when she plays a strategy choice. To capture the requirement in (7), our definition endorses the view that an individual concerns herself with at least her own self; that is, $i \in S(i)$.

Furthermore, given that in Dutta-Sen's Theorem 1 the mechanism designer knows the honesty standard of society, denoted by $S(N) \equiv(S(i))_{i \in N}$, we also need the following information assumption in order to state their result from the perspective of this paper.

Assumption 2 The mechanism designer knows the honesty standard of the society $N$.

Therefore:

Proposition 2 (Dutta-Sen's Theorem 1, 2012) Let Assumption 1 and Assumption 2 be given. Let the honesty standard of society be summarized in $\bar{S}(N)$, where $\bar{S}(i) \equiv N$ for all $i \in N$. If $n \geq 3$ and $F: \Theta \rightarrow X$ is a SCR satisfying $\bar{S}(N)$-partial-honesty monotonicity for the standard $\bar{S}(N)$ and no veto-power, then it is partially-honestly Nash implementable.

It follows from Corollary 1 and Corollary 2 that any SCR is $\bar{S}(N)$-partial-honesty monotonic whenever the honesty standard of society is such that every individual considers truthful only messages that encode the whole truth about preferences of individuals in society, that is, $\bar{S}(i)=N$ for all $i \in N$.

That is a particular kind of honesty standards of individuals but there is no reason to restrict attention to such standards. Thus, in what follows, we are interested in understanding the kind of honesty standards of individuals which would make it impossible for the mechanism designer to circumvent the limitations imposed by Maskin monotonicity. To this end, let us introduce the following notion of standards of honesty of a society.

Definition 11 Given a society $N$ involving at least two individuals, an honesty standard of this society is said to be non-connected if and only if for all $i \in N, i \notin S(j)$ for some $j \in N$.

Given that the honesty standard of individual $i$ includes the individual herself, by definition of $S(i)$, the honesty standard of society is non-connected whenever every one of its 
members is excluded from the honesty standard of another member of the society. Simply put, members of a society do not concern themselves with the same individual.

It is self-evident that the kind of honesty standards in Dutta-Sen's theorem are not non-connected because every individual of the society is interested in telling the truth about the whole society. As another example of honesty standards of a society that are not nonconnected, consider a three-individual society where individual 1 concerns herself with herself and with individual 2 (that is, $S(1)=\{1,2\}$ ), individual 2 concerns herself with everyone (that is, $S(2)=\{1,2,3\})$ and, finally, individual 3 concerns herself with herself and with individual 1 (that is, $S(3)=\{1,3\}$ ). The honesty standard of this three-individual society is not non-connected because everyone concerns themselves with individual 1.

Moreover, it is not necessarily true that every non-connected honesty standard of society implies that every individual honesty standard be of the form $S(i) \neq N$, as we demonstrate with the next example. Consider a three-individual society where individual 1 is concerned only with herself (that is, $S(1)=\{1\}$ ), individual 2 with everyone (that is, $S(2)=\{1,2,3\}$ ) and individual 3 with herself and with individual 2 (that is, $S(3)=\{2,3\}$ ). The honesty standard of this society is non-connected given that individual 2 and individual 3 are both excluded from the honesty standard of individual 1 and individual 1 is excluded from the honesty standard of individual 3.

As is the case here, the above definition is a requirement for the honesty standard of a society that is sufficient for $S(N)$-partial-honesty monotonicity to be equivalent to Maskin monotonicity when two further assumptions are satisfied. The first assumption requires that the family $\mathcal{H}$ includes singletons. This requirement is innocuous given that the mechanism designer cannot exclude any individual from being partially-honest purely on the basis of Assumption 1.

The second requirement is that the set of states $\Theta$ takes the structure of the Cartesian product of allowable independent characteristics for individuals. More formally, the domain $\Theta$ is said to be independent if it takes the form

$$
\Theta=\prod_{i \in N} \Theta_{i}
$$

where $\Theta_{i}$ is the domain of allowable independent characteristics for individual $i$, with $\theta_{i}$ as a typical element. A typical example of an independent domain is that each $\Theta_{i}$ simply represents the domain of the preference orderings over $X$ of individual $i$ and so the domain of the profiles of all individuals' preference orderings on $X$ has the structure of the Cartesian product. In such a case, in a state $\theta=\left(\theta_{i}\right)_{i \in N}$, individual $i$ 's preference ordering over $X$ depends solely on individual $i$ 's independent characteristic $\theta_{i}$ rather than on the profile $\theta$.

The latter two requirements and the requirement that the honesty standard of society 
needs to be non-connected are jointly sufficient for $S(N)$-partial-honesty monotonicity to imply Maskin monotonicity. This can be seen as follows:

Consider a two-individual society where $\Theta$ is the set of states and $X$ is the set of outcomes available to individuals. Let $S(i)$ be the honesty standard of individual $i=1,2$. Consider an outcome $x$ and a state $\theta$ such that $x$ is an $F$-optimal outcome at $\theta$. Consider any other state $\theta^{\prime}$ such that individuals' preferences change in a Maskin monotonic way around $x$ from $\theta$ to $\theta^{\prime}$. Maskin monotonicity says that $x$ must continue to be an $F$-optimal outcome at $\theta^{\prime}$. To avoid trivialities, let us focus on the case that $\theta \neq \theta^{\prime}$, which means that $R_{N}(\theta) \neq R_{N}\left(\theta^{\prime}\right)$, given that we identify states with preference profiles.

If every individual were concerned with the whole society, we could never invoke (the contrapositive of) $S(N)$-partial-honesty monotonicity to conclude that $x$ should remain $F$ optimal at $\theta^{\prime}$ because $R_{N}(\theta) \neq R_{N}\left(\theta^{\prime}\right)$. Furthermore, consider the case that individual 1 concerns herself with only herself, that is, $S(1)=\{1\}$, while individual 2 concerns herself with the whole society, that is, $S(2)=\{1,2\}$. Reasoning such as the one just used shows that $S(N)$-partial-honesty monotonicity cannot be invoked if $R_{1}(\theta) \neq R_{1}\left(\theta^{\prime}\right)$. The argument for honesty standards of the form $S(1)=\{1,2\}$ and $S(2)=\{2\}$ is symmetric. Thus, the only case left to be considered is the one in which everyone concerns themselves with only themselves, that is, $S(i)=\{i\}$ for $i=1,2$. In this situation, the honesty standard of society is reduced to the non-connected one. Note that the standards considered earlier were not non-connected.

Suppose that preferences of individual 1 are identical in the two states, that is, $R_{1}(\theta)=$ $R_{1}\left(\theta^{\prime}\right)$. To conclude that $x$ should be $F$-optimal at $\theta^{\prime}$ by invoking $S(N)$-partial-honesty monotonicity we need to find individual 1 in the family $\mathcal{H}$. The argument for the case $R_{2}(\theta)=R_{2}\left(\theta^{\prime}\right)$ is symmetric. Thus, if $R_{i}(\theta)=R_{i}\left(\theta^{\prime}\right)$ for one of the individuals, the requirement that the singleton $\{i\}$ is an element of $\mathcal{H}$ is needed for the completion of the argument.

Suppose that preferences of individuals are not the same in the two states, that is, $R_{i}(\theta) \neq R_{i}\left(\theta^{\prime}\right)$ for every individual $i$, though they have changed in a Maskin monotonic way around $x$ from the state $\theta$ to $\theta^{\prime}$. In this case, one cannot directly reach the conclusion of Maskin monotonicity by invoking $S(N)$-partial-honesty monotonicity. One way to circumvent the problem is to be able to find a feasible state $\theta^{\prime \prime}$ with the following properties: i) individuals' preferences change in a Maskin monotonic way around $x$ from $\theta$ to $\theta^{\prime \prime}$ and $R_{i}(\theta)=R_{i}\left(\theta^{\prime \prime}\right)$ for an individual $i$, and ii) individuals' preferences change in that way around $x$ from $\theta^{\prime \prime}$ to $\theta^{\prime}$ and $R_{j}\left(\theta^{\prime}\right)=R_{j}\left(\theta^{\prime \prime}\right)$ for individual $j \neq i$. A domain $\Theta$ that assures the existence of such a state is the independent domain.

Even if one were able to find such a state $\theta^{\prime \prime}$ by requiring an independent product structure of $\Theta$, one could not invoke $S(N)$-partial-honesty monotonicity to conclude that $x$ 
must continue to be an $F$-optimal outcome at $\theta^{\prime}$ whenever the family $\mathcal{H}$ did not have the appropriate structure. This can be seen as in the following argument.

Suppose that $\Theta$ is an independent domain. Then, states take the form of profiles of individuals' characteristics, that is, $\theta=\left(\theta_{1}, \theta_{2}\right)$ and $\theta^{\prime}=\left(\theta_{1}^{\prime}, \theta_{2}^{\prime}\right)$. Moreover, the characteristic of individual $i$ in one state is independent from the characteristic of the other individual. That is, $R_{i}(\theta)=R_{i}\left(\theta_{i}\right)$ and $R_{i}\left(\theta^{\prime}\right)=R_{i}\left(\theta_{i}^{\prime}\right)$ for every individual $i$. The product structure of $\Theta$ assures that the states $\left(\theta_{1}, \theta_{2}^{\prime}\right)$ and $\left(\theta_{1}^{\prime}, \theta_{2}\right)$ are both available and each of them has the properties summarized above.

Next, suppose that the family $\mathcal{H}$ has a structure given by $\{\{1\},\{1,2\}\}$. One can invoke $S(N)$-partial-honesty monotonicity for $H=\{1\}$ to obtain that $x$ is one of the outcomes chosen by the SCR $F$ at $\left(\theta_{1}, \theta_{2}^{\prime}\right)$ when the state changes from $\theta$ to $\left(\theta_{1}, \theta_{2}^{\prime}\right)$, but he cannot conclude that $x$ remains also $F$-optimal at $\theta^{\prime}$ when it changes from $\left(\theta_{1}, \theta_{2}^{\prime}\right)$ to $\theta^{\prime}$. The reason is that $S(N)$-partial-honesty monotonicity cannot be invoked again for the case $H=\{2\}$ because the structure of the family $\mathcal{H}$ does not contemplate such a case. The argument for the case that $\mathcal{H}$ takes the form $\{\{2\},\{1,2\}\}$ is symmetric. Thus, each of our requirements is indispensable, and jointly they lead to the following conclusion:

Theorem 4 Let $N$ be a society involving at least two individuals, $\Theta$ be an independent domain and $\mathcal{H}$ include singletons. Suppose that the honesty standard of the society, denoted by $S(N)$, is non-connected. Then, $S(N)$-partial-honesty monotonicity is equivalent to Maskin monotonicity.

Proof. Let $n \geq 2, \Theta$ be an independent domain and $\mathcal{H}$ include singletons. Let $S(N)$ be a non-connected honesty standard of $N$. One can see that Maskin monotonicity implies $S(N)$-partial-honesty monotonicity.

For the converse, consider any SCR $F: \Theta \rightarrow X$ satisfying $S(N)$-partial-honesty monotonicity. Consider any $x \in X$ and any state $\theta \in \Theta$ such that $x$ is an F-optimal outcome at $\theta$. Moreover, consider any state $\theta^{\prime}$ such that individuals' preferences change in a Maskin monotonic way around $x$ from $\theta$ to $\theta^{\prime}$, that is,

$$
\text { for all } i \in N \text { and all } x^{\prime} \in X: x R_{i}(\theta) x^{\prime} \Longrightarrow x R_{i}\left(\theta^{\prime}\right) x^{\prime} \text {. }
$$

We show that $x$ remains $F$-optimal at $\theta^{\prime}$.

If characteristics of individuals in the honesty standard of individual $i \in N$ are identical in the two states, that is, $R_{S(i)}(\theta)=R_{S(i)}\left(\theta^{\prime}\right), S(N)$-partial-honesty monotonicity for the case $H=\{i\}$ assures that $x$ is still $F$-optimal at $\theta^{\prime}$. Thus, let us consider the case $R_{S(i)}(\theta) \neq$ $R_{S(i)}\left(\theta^{\prime}\right)$ for every individual $i \in N$.

To economize notation, for any subset $K$ of $N$, write $K_{C}$ for the complement of $K$ in $N$. Therefore, for any non-empty subset $K$ of $N$, we can write any non-trivial combination 
of the states $\theta$ and $\theta^{\prime}$ as $\left(\theta_{K}, \theta_{K_{C}}^{\prime}\right)$, where it is understood that $\theta_{K}$ is a list of characteristics of individuals in $K$ at the state $\theta$ and $\theta_{K_{C}}^{\prime}$ is a list of characteristics of individuals in $K_{C}$ at $\theta^{\prime}$. Note that any state that results by that combination is available in $\Theta$ because of its product structure.

Given that the honesty standard of society is non-connected, there must be an individual $j(1) \in N$ who does not concern herself with the whole society, that is, $S(j(1)) \neq N$. Consider the state

$$
\left(\theta_{K(1)}, \theta_{K(1)_{C}}^{\prime}\right) \text { where } K(1) \equiv S(j(1))
$$

and call it $\theta^{1}$. By construction, individuals' preferences change in a Maskin monotonic way around $x$ from $\theta$ to $\theta^{1}$ and, moreover, $\theta_{K(1)}=\theta_{K(1)}^{1}$. $S(N)$-partial-honesty monotonicity for the case $H=\{j(1)\}$ assures that the $x$ remains an $F$-optimal outcome at $\theta^{1}$.

If there is an individual $i \in N \backslash\{j(1)\}$ who is not concerned with any of the individuals in the honesty standard of individual $j(1)$, that is, the intersection $S(i) \cap S(j(1))$ is empty, then $S(N)$-partial-honesty monotonicity for the case $H=\{i\}$ assures that $x$ is still $F$ optimal at $\theta^{\prime}$. This is because, by construction, individuals' preferences change in a Maskin monotonic way around $x$ from $\theta^{1}$ to $\theta^{\prime}$ and $\theta_{S(i)}^{1}=\theta_{S(i)}^{\prime}$.

Thus, consider any individual $j(2) \in N \backslash\{j(1)\}$, and denote by $K(2)$ the set of individuals with whom individual $j(1)$ and individual $j(2)$ are jointly concerned according to their individual honesty standards. Furthermore, consider the state

$$
\left(\theta_{K(2)}, \theta_{K(2)_{C}}^{\prime}\right) \text { where } K(2) \equiv K(1) \cap S(j(2)),
$$

and call it $\theta^{2}$. By construction, individuals' preferences change in a Maskin monotonic way around $x$ from $\theta^{1}$ to $\theta^{2}$ and, moreover, $\theta_{S(j(2))}^{1}=\theta_{S(j(2))}^{2}$. $S(N)$-partial-honesty monotonicity for the case $H=\{j(2)\}$ assures that $x$ remains an $F$-optimal outcome at $\theta^{2}$.

If there is an individual $i \in N \backslash\{j(1), j(2)\}$ who is not concerned with any of the individuals with whom individuals $j(1)$ and $j(2)$ are jointly concerned, $S(N)$-partial-honesty monotonicity for the case $H=\{i\}$ assures that $x$ is also $F$-optimal at $\theta^{\prime}$. This is because, by construction, individuals' preferences change in a Maskin monotonic way around $x$ from $\theta^{2}$ to $\theta^{\prime}$ and $\theta_{S(i)}^{2}=\theta_{S(i)}^{\prime}$.

Thus, consider any individual $j(3) \in N \backslash\{j(1), j(2)\}$, and denote by $K(3)$ the set of individuals with whom individuals $j(1), j(2)$ and $j(3)$ are jointly concerned according to their individual honesty standards. Furthermore, consider the state

$$
\left(\theta_{K(3)}, \theta_{K(3)_{C}}^{\prime}\right) \text { where } K(3) \equiv K(2) \cap S(j(3)),
$$

and call it $\theta^{3}$. By construction, individuals' preferences change in a Maskin monotonic way 
around $x$ from $\theta^{2}$ to $\theta^{3}$ and, moreover, $\theta_{S(j(3))}^{2}=\theta_{S(j(3))}^{3} . S(N)$-partial-honesty monotonicity for the case $H=\{j(3)\}$ assures that $x$ remains an $F$-optimal outcome at $\theta^{3}$.

As above, if there is an individual $i \in N \backslash\{j(1), j(2), j(3)\}$ who is not concerned with any of the individuals with whom individuals $j(1), j(2)$ and $j(3)$ are jointly concerned, $S(N)$-partial-honesty monotonicity for the case $H=\{i\}$ assures that $x$ remains also $F$-optimal at $\theta^{\prime}$, because, by construction, individuals' preferences change in a Maskin monotonic way around $x$ from $\theta^{3}$ to $\theta^{\prime}$ and $\theta_{S(i)}^{3}=\theta_{S(i)}^{\prime}$. And so on.

Since the society $N$ is a finite set and the above iterative reasoning is based on its cardinality, we are left to show that it must stop at most after $n-1$ iterations.

To this end, suppose that we have reached the start of the $n-1$ th iteration. Thus, consider any individual $j(n-1) \in N$, with $j(n-1) \neq j(r)$ for $r=1, \cdots, n-2$, and denote by $K(n-1)$ the set of individuals with whom individuals $j(1), j(2), \cdots, j(n-2)$ and $j(n-1)$ are jointly concerned according to their individual honesty standards. Furthermore, consider the state

$$
\left(\theta_{K(n-1)}, \theta_{K(n-1)_{C}}^{\prime}\right) \text { where } K(n-1) \equiv K(n-2) \cap S(j(n-1)),
$$

and call it $\theta^{n-1}$. As above, by construction, individuals' preferences change in a Maskin monotonic way around $x$ from $\theta^{n-2} \equiv\left(\theta_{K(n-2)}, \theta_{K(n-2)_{C}}^{\prime}\right)$ to $\theta^{n-1}$ and, moreover, $\theta_{S(j(n-1))}^{n-2}=$ $\theta_{S(j(n-1))}^{n-1} . S(N)$-partial-honesty monotonicity for the case $H=\{j(n-1)\}$ assures that $x$ is an $F$-optimal outcome at $\theta^{n-1}$.

At this stage there is only one individual in $N$ who is left to be considered. Call her $j(n)$. Suppose that this individual is concerned with one of the individuals for whom individuals $j(1), j(2), \cdots, j(n-2)$ and $j(n-1)$ are jointly concerned. In other words, suppose that the intersection $K(n-1) \cap S(j(n))$ is non-empty. Then, the whole society concerns itself with one of its member, and this contradicts the fact that the honesty standard of society is non-connected. Therefore, it must be the case that individual $j(n)$ is not concerned with any of the individuals with whom individuals $j(1), j(2), \cdots, j(n-2)$ and $j(n-1)$ are jointly concerned according to their individual honesty standards. $S(N)$-partial-honesty monotonicity for the case $H=\{j(n)\}$ assures that $x$ remains also $F$-optimal at $\theta^{\prime}$ given that, by construction, individuals' preferences change in a Maskin monotonic way around $x$ from $\theta^{n-1}$ to $\theta^{\prime}$ and $\theta_{S(j(n))}^{n-1}=\theta_{S(j(n))}^{\prime}$.

The iterative reasoning would stop at the $r$ th $(<n-1)$ iteration if there were an individual $i \in N \backslash\{j(1), \cdots, j(r)\}$ who was not concerned with any of the individuals in $K(r)$, that is, if the intersection $S(i) \cap K(r)$ were empty. If that were the case, then the desired conclusion could be obtained by invoking $S(N)$-partial-honesty monotonicity for $H=\{i\}$ because, by construction, it would hold that individuals' preferences change in a Maskin 
monotonic way around $x$ from $\theta^{r}$ to $\theta^{\prime}$ and that $\theta_{S(i)}^{r}=\theta_{S(i)}^{\prime}$.

In light of Theorem 2 and Maskin's theorem, the main implications of Theorem 4 can be formally stated as follows:

Corollary 3 Let $N$ be a society involving at least two individuals, $\Theta$ be an independent domain and $\mathcal{H}$ include singletons. Suppose that the honesty standard $S(N)$ of the society is non-connected. Let Assumption 1 be given. Then, a SCR $F: \Theta \rightarrow X$ is Maskin monotonic if it is partially-honestly Nash implementable.

Corollary 4 Let $N$ be a society involving at least three individuals, $\Theta$ be an independent domain and $\mathcal{H}$ include singletons. Suppose that the honesty standard $S(N)$ of the society is non-connected. Let Assumption 1 be given. Then, a SCR $F: \Theta \rightarrow X$ satisfying no veto-power is partially-honestly Nash implementable if and only if it is Maskin monotonic.

Remark 3 In a related but not identical setting, Kartik and Tercieux (2012) study Nash implementation problems where agents can choose to provide evidence as part of their strategies. In this setup, they show that any social choice function satisfying a weaker variant of Maskin monotonicity, called evidence-monotonicity, and no veto-power is Nash implementable. In an environment where there are partially-honest individuals, they show that even small intrinsic costs of lying create a substantial wedge between evidence-monotonicity and Maskin monotonicity, in the sense that every social choice function is evidence-monotonic. Under the assumptions of Theorem 4 and suitable specifications which resemble those of Example 2 in Kartik and Tercieux (2012; p. 333), one can show that this wedge disappears when participants are allowed/forced to produce partial evidence of the true state according to a non-connected (evidence) standard $S(N){ }^{4}$

\footnotetext{
${ }^{4}$ To see it, let us suppose that individuals have separable preferences in the sense of Kartik and Tercieux (2012; p. 238). That is, suppose that each agent's (extended) preference ordering $R_{i}(\theta)$ over the outcomeevidence space $X \times E_{i}$ is represented by a utility function of the form $U_{i}\left(x, e_{i}, \theta\right)=u_{i}(a, \theta)-c_{i}\left(e_{i}, \theta\right)$, where $c_{i}\left(e_{i}, \theta\right)$ represents agent $i$ 's cost of producing evidence $e_{i}$. Fix any $S(N)$ and let the domain $\Theta$ be independent. For each individual $i$, let the evidence space be $E_{i}=\prod_{j \in S(i)} \Theta_{j}$. Fix any set $H$. For each $h \in H$, let the cost function be $c_{h}\left(\theta, \theta^{\prime}\right)=0$ if $R_{S(h)}(\theta)=R_{S(h)}\left(\theta^{\prime}\right)$, otherwise, $c_{h}\left(\theta, \theta^{\prime}\right)=\varepsilon>0$, where $\varepsilon$ can be arbitrarly small. For each $i \notin H$, let $c_{i}\left(\theta, \theta^{\prime}\right)=0$ for every $\theta$ and $\theta^{\prime}$. This structure implies that the set of the least-evidence cost for $h \in H$ given the pair $(x, \theta)$ is $E_{h}^{\ell}(x, \theta)=\left\{\theta_{S(h)}\right\}$ while it is $E_{i}^{\ell}(x, \theta)=E_{i}$ for every $i \notin H$. Let the evidence function of invididual $h \in H$ be $e_{h}^{*}(\theta)=\left\{R_{S(h)}(\theta)\right\}$ for every $\theta \in \Theta$. Under these specifications, one can now see from the proof of Theorem 4 that evidence-monotonicity (stated for each $H \in \mathcal{H}$ ) is equivalent to Maskin monotonicity.
} 


\section{Concluding remarks}

The assumption that the mechanism designer knows the honesty standard of society is often not met in reality, although it may be plausible in societies with a small number of individuals in which the mechanism designer knows their sensitivity to honesty. Outside of cases like those, we view as more plausible the assumption that the mechanism designer only knows the types of honesty standards shared by individuals. Does the conclusion of Theorem 4 change in this case? The answer is no. After all, if individuals are honesty-sensitive, the mechanism designer can test for connectedness of their honesty standards. If the test fails, it would be in vain for him to attempt to Nash implement any SCR that is not Maskin monotonic. The reason for it is easy to identify: the fact that he solely knows that the honesty standard of society is non-connected can only make implementation harder than if the actual non-connected honesty standards of participants were known.

Theorem 4 is derived on the basis that in every state a strategy choice of an individual is truthful if it encodes information of individuals' preferences consistent with that state for members of society in her honesty standard. This implies that if we arrange agents in a directed circle and ask them to report their own preferences and those of their successors in the circle, and the honesty standard of every individual includes herself and her successors, ${ }^{5}$ then this 'simpler' mechanism would impair the ability of the mechanism designer to escape the limitations imposed by Maskin monotonicity. Then, a natural question that arises immediately is: Under what conditions would the positive result of Dutta and Sen (2012) be restored? We answer this question in a companion paper (Lombardi and Yoshihara, 2016a) and it is as follows: The mechanism designer who knows that $\alpha(\geq 1)$ members of society have a taste for honesty can expect to do well if no participant has a veto-power by structuring communication with participants in a way that each of them reports her own preference and those of other $n-\alpha$ successors who are in her honesty standard.

Postlewaite and Schmeidler (1986), Palfrey and Srivastava (1989) and Jackson (1991) have shown that Maskin's theorem can be generalized to Bayesian environments. A necessary condition for Bayesian Nash implementation is Bayesian monotonicity. In a Bayesian environment involving at least three individuals, Bayesian monotonicity combined with no veto-power is sufficient for Bayesian Nash implementation provided that a necessary condition called closure and the Bayesian incentive compatibility condition are satisfied (Jackson, 1991). Although the implementation model developed in this paper needs to be modified to handle Bayesian environments, we believe a similar equivalence result holds in those en-

\footnotetext{
${ }^{5}$ In an environment in which knowledge is dispersed, how individuals will interact with the mechanism designer is a natural starting point when it comes to Nash implementing a SCR. A particular kind of communication is, as in Dutta and Sen's (2012) Theorem 1, to ask participants to report preferences of the entire society. However, there is no reason to restrict attention to such schemes.
} 
vironments for suitably defined communication schemes (on this point, see Lombardi and Yoshihara, 2013; section 5). This subject is left for future research.

\section{References}

Abreu, D., Sen, A., 1990. Subgame perfect implementation: a necessary and almost sufficient condition, J. Econ. Theory 50, 285-299.

Abreu, D., Sen, A., 1991. Virtual implementation in Nash equilibrium, Econometrica 59, 997-1021.

Corchón, L.C., Herrero, C., 2004. A decent proposal, Spanish Econ. Rev. 6, 107-125.

Dutta, B., Sen, A., 1991. A necessary and sufficient condition for two-person Nash implementation, Rev. Econ. Stud. 58, 121-128.

Dutta, B., Sen, A., 2012. Nash implementation with partially honest individuals, Games Econ Behav 74, 154-169.

Jackson, M.O., 1991. Bayesian implementation, Econometrica 59, 461-477.

Jackson, M.O., 1992. Implementation in Undominated Strategies: A Look at Bounded Mechanisms, Rev. Econ. Stud. 59, 757-775.

Jackson, M.O., 2001. A crash course in implementation theory, Soc. Choice Welfare 18, 655-708.

Kalai, E., Ledyard, J.O., 1998. Repeated Implementation, J. Econ. Theory 83, 308-317.

Kartik, N., Tercieux, O., 2012. Implementation with evidence, Theor. Econ. 7, 323-355.

Kartik, N., Tercieux, O., Holden, R., 2014. Simple mechanism and preference for honesty, Games Econ. Behav. 83, 284-290.

Lee, J., Sabourian, H., 2011. Efficient Repeated Implementation, Econometrica 79, 19671994.

Lombardi, M., Yoshihara, N., 2013. A full characterization of Nash implementation with strategy space reduction, Econ. Theory 54, 131-151.

Lombardi, M., Yoshihara, N., 2016a. Treading a Fine Line: (Im)possibilities for Nash Implementation with Partially-honest Individuals, IER DP series A, n. 651, Hitotsubashi Univ.

Lombardi, M., Yoshihara, N., 2016b. Natural implementation with semi-responsible agents in pure exchange economies, forthcoming in International J. Game Theory. 
Lombardi, M., Yoshihara, N., 2016c. Partially-honest Nash implementation: a full characterization, mimeo.

Maskin, E., 1999. Nash equilibrium and welfare optimality, Rev. Econ. Stud. 66, 23-38.

Maskin, E., Sjöström, T., 2002. Implementation theory, in: K. Arrow, A.K. Sen, K. Suzumura (Eds), Handbook of Social Choice and Welfare, Elsevier Science, Amsterdam, pp. $237-288$.

Matsushima, H., 1988. A new approach to the implementation problem, J. Econ. Theory 45, $128-144$.

Matsushima, H., 2008a. Behavioral aspects of implementation theory, Econ. Letters 100, 161-164.

Matsushima, H., 2008b. Role of honesty in full implementation, J. Econ. Theory 139, 353359.

Mezzetti, C., Renou, L., 2016. Repeated Nash Implementation. To appear in Theoretical Economics.

Moore, J., Repullo, R., 1988. Subgame perfect implementation, Econometrica 56, 1191-1220.

Moore, J., Repullo, R., 1990. Nash implementation: A full characterization, Econometrica $58,1083-1100$.

Ortner, J., 2015. Direct implementation with partially honest individuals, Games Econ. Behav. 90, 1-16.

Palfrey, T., Srivastava, S., 1989. Implementation with incomplete information in exchange economies, Econometrica 57, 115-134.

Palfrey, T., Srivastava, S., 1991. Nash-implementation using undominated strategies, Econometrica 59, 479-501.

Postlewaite, A., Schmeidler, D., 1986. Implementation in differential information economies, J. Econ. Theory 39, 14-33.

Saporiti, A., 2014. Securely implementable social choice rules with partially honest agents, J. Econ. Theory 154, 216-228.

Serrano, R., 2004. The theory of implementation of social choice rules, SIAM Review 46, $377-414$.

Sjöström, T., 1991. On the necessary and sufficient conditions for Nash implementation, Soc. Choice Welfare 8, 333-340. 